\title{
Metataxonomic sequencing reveals compositional shifts within the denture-associated microbiome in pneumonia
}

Joshua Alexander Twigg ( $\nabla$ denjtw@leeds.ac.uk)

Leeds Dental Institute https://orcid.org/0000-0002-4567-7854

Ann Smith

University of the West of England Bristol

Clotilde Haury

Cardiff University

Melanie J Wilson

Cardiff University

Jonathan Lees

Cardiff University

Mark Waters

Cardiff University

David W Williams

Cardiff University

Research

Keywords: Oral microbiome, pneumonia, 16S rRNA gene, denture, saliva

Posted Date: February 10th, 2020

DOI: https://doi.org/10.21203/rs.2.23022/v1

License: (1) This work is licensed under a Creative Commons Attribution 4.0 International License. Read Full License 


\section{Abstract}

Background Bacterial pneumonia affects a disproportionate number of the elderly in the UK, with substantial morbidity and mortality. Mounting evidence implicates removable dentures as a potential nidus for respiratory pathogens to form a reservoir which could seed colonisation and infection of respiratory tissues in susceptible individuals. However, research evaluating the denture-associated microbiome in patients with an active diagnosis of pneumonia is lacking. The aim of this study was to characterise denture-associated oral bacterial communities by metataxonomic sequencing of 16S rRNA genes. The prevalence of antimicrobial resistance among two representative pathogenic species Staphylococcus aureus and Pseudomonas aeruginosa was also assessed. Finally, the role of salivary cytokines as diagnostic biomarkers was explored.

Results There were significant shifts observed in species composition, diversity and richness in the denture-associated microbiome of pneumonia patients. Importantly, the relative abundance of putative respiratory pathogens in the dentureassociated microbiota of pneumonia patients was significantly increased compared with respiratorily healthy care home residents. The magnitude of this increase was approximately three-fold in denture-associated bacterial communities compared with other oral sites examined. Antimicrobial resistance was equivocal between microbes isolated from both participant cohorts, highlighting the potential for oral biofilms to protect microbes from systemic antimicrobial therapy. While salivary cytokine profiles did not correlate with pneumonia status, the concentration of IL- 6 and IL-8 positively correlated with the relative abundance of putative respiratory pathogens on denture surfaces.

Conclusions This is the first study to directly examine compositional shifts in the denture-associated oral microbiome in respiratory infection, providing a basis for disentangling potential causal relationships.

\section{Background}

Lower respiratory tract infections, including pneumonia, are the fourth leading cause of death worldwide, and the most common cause of death due to infectious disease ${ }^{1}$. Globally, pneumonia has a bimodal distribution of incidence, affecting the very young and elderly. However, in the United Kingdom, much of Europe and the USA, pneumonia demonstrates a predilection for the elderly, with a ten-fold increase in pneumonia cases in patients over 65 years of age ${ }^{2}$ and $85 \%$ of pneumonia-related deaths occurring in individuals over the age of 60 years ${ }^{3}$.

The term pneumonia describes a clinical phenotype of acute inflammation in the lower respiratory tract ${ }^{4}$ which does not necessarily reflect an infectious aetiology. However, the vast majority of pneumonias occur secondary to microbial infection, which may be viral, bacterial, fungal, or polymicrobial ${ }^{5}$. In the UK and much of Europe, pneumonia is most frequently bacterial in aetiology ${ }^{6}$. Diagnosis of pneumonia is challenging due to the non-specific clinical signs and symptoms associated with the disease. Determining a microbial aetiology is confounded by the difficulty in obtaining a representative sample free from contaminating microorganisms originating in uninfected regions of the respiratory tissues or oropharynx, and the inability to distinguish microbes colonising the respiratory tissues from infective species ${ }^{7}$.

A burgeoning body of research has revealed an association between changes in oral microbial communities and respiratory infection in susceptible individuals ${ }^{8,9,10,11}$. This is most clearly supported in ventilator-associated pneumonia (VAP) which can affect mechanically ventilated intensive care patients. Here, an increase in the relative abundance of putative respiratory pathogens (PRPs) in dental plaque occurs following intubation of patients in intensive care, with a subsequent reversal of this compositional perturbation following extubation ${ }^{12,13}$. Further, a recent systematic review found evidence supporting the effectiveness of oral care to reduce VAP, although the effect size was modest and the overall quality of evidence available was low ${ }^{14}$. Similarly, a number of researchers have recovered PRPs from denture surfaces ${ }^{15,16,17}$, while enhanced oral care, including denture care, has been found to reduce the incidence of pneumonia among long-term care facility residents ${ }^{18}$. The presence of an endotracheal tube offers a direct conduit to the lungs and necessitates open mouth posture, facilitating the acquisition of exogenous microorganisms; bypassing the host immune system and enabling translocation to the respiratory 
tissues ${ }^{19}$. That a similar relationship appears to exist between the denture-associated oral microbiota and respiratory infection suggests that the presence of an artificial biomaterial surface may itself promote colonisation by PRPs, forming a reservoir that can seed infection of the respiratory tissues in susceptible individuals.

Despite indirect evidence suggesting that the oral microbial communities of denture-wearing individuals may contribute to pneumonia risk, direct support for a mechanistic role for denture biomaterial surfaces in promoting respiratory infection is lacking. Therefore, this study aimed to compare the community composition of denture-associated oral bacteria in patients with a clinical diagnosis of pneumonia with respiratorily healthy care home residents. The potential role of salivary cytokines as biomarkers for both pneumonia status and oral PRP bioburden was also evaluated.

\section{Results}

\section{Participant demographics and clinical characteristics}

Participant demographic information is summarised in Table 1. Pneumonia patients were significantly younger than care home residents (Mean difference 4 years, $p=0.0006$ ). All pneumonia patients received antibiotic therapy (intravenous amoxicillin and clarithromycin, $n=15$; other, $n=11$ ), while only 4 of the 35 care home residents included had received antibiotics in the preceding 6 months. Otherwise there were no significant differences observed between participant cohorts.

Table 1: Summary participant information

Care home residents $(\mathrm{n}=35)$

\begin{tabular}{lll}
\hline Mean Age (S.D.) & $88(7.6)$ & $84(8.2)$ \\
\hline Gender (\%) & $15 \%$ male & $15 \%$ male \\
\hline Antibiotics in last 90 days (\%) & $15 \%$ & $100 \%$ \\
\hline Smoking History (\%) & $15 \%$ current smokers & $8 \%$ current smokers \\
& $54 \%$ ex-smokers & $65 \%$ ex-smokers \\
& $31 \%$ never smoked & $27 \%$ never smoked \\
\hline
\end{tabular}

Mean Charlson Comorbidity Index\#

(S.D.)

\begin{tabular}{|c|c|c|c|c|c|c|}
\hline \multirow[t]{2}{*}{ Mean DMFT score* (S.D.) } & Decayed & Missing & Filled & Decayed & Missing & Filled \\
\hline & $\begin{array}{l}1.6 \\
(2.00)\end{array}$ & $25.0(5.33)$ & $2.3(4.38)$ & $1.8(1.48)$ & $24.3(5.77)$ & $3.6(3.06)$ \\
\hline \multirow[t]{3}{*}{ Complete or Partial Denture (\%) } & \multicolumn{3}{|c|}{$62.9 \%$ Complete } & \multicolumn{3}{|c|}{$59.6 \%$ Complete } \\
\hline & \multicolumn{3}{|c|}{$27.1 \%$ Partial } & \multicolumn{3}{|c|}{ 15.4\% Partial } \\
\hline & \multicolumn{3}{|c|}{ (10\% no denture in one arch) } & \multicolumn{3}{|c|}{ ( $25 \%$ no denture in one arch) } \\
\hline \multirow[t]{2}{*}{ Acrylic or Cobalt Chromium Denture (\%) } & \multicolumn{3}{|c|}{ 91.4\% Acrylic } & \multicolumn{3}{|c|}{ 92.3\% Acrylic } \\
\hline & \multicolumn{3}{|c|}{ 8.6\% Cobalt chromium } & \multicolumn{3}{|c|}{ 7.7\% Cobalt chromium } \\
\hline Mean Denture Cleanliness Index ${ }^{+}$(S.D.) & \multicolumn{3}{|l|}{$1.8(1.11)$} & \multicolumn{3}{|l|}{$1.6(1.19)$} \\
\hline Mean Newton Index $\$$ (S.D.) & \multicolumn{3}{|l|}{$0.9(0.53)$} & \multicolumn{3}{|l|}{$1.1(0.80)$} \\
\hline
\end{tabular}


\# Charlson Comorbidity Index scores a number of physiological measures and diseases to provide estimate of 10-year survival. The Maximum score (highest mortality risk) is 33. A score of 7 or greater indicates a predicted 10-year survival rate of $0 \%$.

* A DMFT score is indicative of Decayed, Missing, Filled Teeth. Absence of a tooth, or the presence of any dental restoration or caries scores 1 point. The maximum score is 28 . Wisdom teeth were not included in this score.

+ Denture Cleanliness Index scores denture cleanliness from 0 (pristine denture surfaces) to 4 (damaged dentures).

\$ Newton Index scores palatal inflammation from 0 (normal, healthy mucosa) to 3 (grossly erythematous, swollen mucosa).

\section{Culture isolation and antimicrobial susceptibility testing of target microorganisms}

Candida was recovered from almost $90 \%$ of patients' oral cavities, an unsurprising finding given the known high prevalence of this yeast in denture-wearing individual ${ }^{29}$. Both $S$. aureus and $P$. aeruginosa, two pathogens frequently associated with respiratory infection and a range of healthcare associated infections, were recovered from the oral cavities of individuals in both cohorts (Table 2). There was no statistically significant difference between recovery rates between patients with pneumonia and respiratorily healthy individuals.

Table 2: Recovery rates (\%) of target microorganisms by culture

\begin{tabular}{|c|c|c|c|c|c|c|c|c|c|c|}
\hline & \multirow{2}{*}{$\begin{array}{l}\text { Microbial } \\
\text { species }\end{array}$} & \multicolumn{3}{|c|}{ Candida species } & \multicolumn{3}{|c|}{ Staphylococcus aureus } & \multicolumn{3}{|c|}{ Pseudomonas aeruginosa } \\
\hline & & Tongue & Palate & Denture & Tongue & Palate & Denture & Tongue & Palate & Denture \\
\hline \multirow{3}{*}{$\begin{array}{l}\text { Participant } \\
\text { cohort }\end{array}$} & Overall & 88.5 & 83.7 & 88.0 & 21.0 & 13.4 & 15.8 & 10.1 & 7.2 & 10.1 \\
\hline & Care home & 88.6 & 82.9 & 91.4 & 22.9 & 11.4 & 20.0 & 8.6 & 2.9 & 8.6 \\
\hline & $\begin{array}{l}\text { Respiratory } \\
\text { ward }\end{array}$ & 88.5 & 84.6 & 84.6 & 19.2 & 15.4 & 11.5 & 11.5 & 11.5 & 11.5 \\
\hline
\end{tabular}

Cultured isolates of $S$. aureus and $P$. aeruginosa were tested for susceptibility to a range of relevant antimicrobials (Table 3 ). Resistance rates varied between different antimicrobials, with no clear trend for isolated microbes from pneumonia patients to exhibit increased resistance to b-lactams, although there was greater resistance of $S$. aureus isolates to macrolides.

Table 3: Antimicrobial resistance rates (\%) among cultured isolates of S. aureus 
$\begin{array}{lll}\begin{array}{l}\text { Care Home Residents }(n=22 \\ \text { isolates) }\end{array} & \begin{array}{l}\text { Respiratory Ward Patients } \\ (n=10 \text { isolates })\end{array} & \begin{array}{l}\text { Overall }(n=32 \\ \text { isolates })\end{array}\end{array}$

\begin{tabular}{llll}
\hline Amoxicillin & 27.3 & 0 & 18.8 \\
\hline $\begin{array}{l}\text { Co- } \\
\text { Amoxiclav }\end{array}$ & 0 & 0 & 0 \\
\hline Gentamicin & 4.6 & 0 & 3.1 \\
\hline Fusidic acid & 13.6 & 20.0 & 15.63 \\
\hline Erythromycin & 18.2 & 50.0 & $28.1(3.1$ \\
& & $(10.0$ intermediate $)$ & intermediate $)$ \\
\hline Clindamycin & 13.6 & 50.0 & 25.0 \\
\hline Cefoxitin & 4.6 & 0 & 3.1
\end{tabular}

Table 4: Antimicrobial resistance rates (\%) among cultured isolates of $P$. aeruginosa

\begin{tabular}{llll} 
& $\begin{array}{l}\text { Care Home Residents } \\
(\mathbf{n}=\mathbf{7} \text { isolates })\end{array}$ & $\begin{array}{l}\text { Respiratory Ward Patients } \\
(\mathbf{n = 1 3} \text { isolates })\end{array}$ & Overall $\mathbf{( n = 2 0 ~ i s o l a t e s )}$ \\
\hline Amikacin & $0(57.4$ intermediate $)$ & $7.7(92.3$ intermediate $)$ & $5.0(80.0$ intermediate $)$ \\
\hline Gentamicin & 0 & 0 & 0 \\
\hline Imipenem & $14.3(28.6$ intermediate $)$ & 0 & $5.0(10.0$ intermediate $)$ \\
\hline Pipericillin-Tazobactam & 85.7 & 46.2 & 60.0 \\
\hline Ciprofloxacin & $0(28.6$ intermediate $)$ & 84.6 & $55.0(10.0$ intermediate $)$ \\
\hline Ceftazidime & 85.7 & 0 & 30.0
\end{tabular}

\section{Analysis of metataxonomic sequencing data}

Analysis of metataxonomic sequencing data revealed an increased relative abundance of Enterobacteriaceae in all oral sites of patients with pneumonia. Although there was a trend of increased relative abundance of most PRP species, this did not reach the threshold of statistical significance (Figure 1). However, when the cumulative relative abundance of all PRPs was assessed, there was a significant increase noted in pathogenic bioburden compared with respiratorily health care home residents (Figure 2a). Calculation of the fold-difference in the cumulative relative abundance of PRPs showed that the increase in pathogenic bioburden was especially elevated in denture samples, with a greater than 20-fold increase in PRPs (Figure 2b).

In keeping with these findings, there were significant compositional shifts in the microbial communities, with a decrease in species richness and beta diversity in bacterial communities (Figure 3) measured by Chao2 and Inverse Simpson indices, respectively. The decreased community diversity and species richness were observed in dorsal tongue and denture samples only.

Figure 3 - Chao (upper panel) and Inverse Simpson (lower panel) indices for oral sites in each participant cohort 
Individual data points with representative box plots shown.

Further exploration of bacterial community composition by linear discriminant analysis (LDA) confirmed an increased bioburden of PRP species with a concomitant reduction in typical oral commensals in pneumonia patients (Figure 4).

Figure 4: LeFSe analysis of differential OTU relative abundance between participant cohorts for denture samples

1. Histogram of significantly different Linear Discriminant Analysis scores for samples.

2. Cladogram representing taxonomic relationships of significantly different abundances between cohort samples.

Green indicates taxons with increased abundance in pneumonia patients, red indicates taxons with increased abundance in samples from care home residents. Yellow circles represent taxa which showed no significant differences between cohorts. The diameter of each circle in the cladogram is proportional to the relative abundance of the taxon represented.

\section{Analysis of salivary cytokine profiles}

The level of proinflammatory cytokines assessed in saliva did not significantly differ with pneumonia status (Figure 5). However, further analysis by linear regression revealed a significant association of IL-6 and IL-8 with the cumulative abundance of PRP species in oral (data not shown) and denture (Figure 6) samples. The proportion of PRPs present in denture samples accounted for approximately $30 \%$ of the variability in cytokine level.

\section{Figure 5: Salivary cytokine expression levels $(\mathrm{pg} / \mathrm{ml})$}

Note that the $Y$ axis uses a $\log _{10}$ scale.

Individual points represent each sample measured. Horizontal black line indicates median expression level for each cohort.

Figure 6: Linear regression of salivary cytokine level against cumulative relative abundance of PRP species

$95 \%$ confidence intervals indicated by dashed lines. $\mathrm{P}$ values represent significance following $\mathrm{F}$ test with Bonferroni post-hoc correction.

\section{Discussion}

While there has been mounting interest in exploring artificial biomaterial surfaces in the oral cavity as potential reservoirs of respiratory pathogens, this was the first study to directly explore compositional shifts in the denture-associated oral 
microbiome correlated with pneumonia status; using contemporary molecular techniques to limit selectivity bias. Not only was an increased bioburden of putative respiratory pathogens in individuals with pneumonia found; there was a concurrent loss of species richness and diversity typically associated with a dysbiotic shift in the microbial community. Importantly, these differences were especially pronounced in denture samples, highlighting the role of dentures as a possible nidus for respiratory infection. The potential for salivary cytokines as biomarkers of pneumonia was also assessed. While cytokine levels were highly variable and did not correlate with pneumonia status directly, there were statistically significant associations of the levels of IL-6 and IL- 8 with the total bioburden of respiratory pathogens in denture samples. These markers may therefore offer promise as adjuncts to identify individuals at risk of respiratory infection secondary to oropharyngeal colonisation by PRPs.

In order to reach, colonise and infect the lungs, bacteria must either pass from an external source through the oral cavity, or intrinsically through the gastrointestinal tract ${ }^{30}$. Thus, the relationship seen between the oral microbiome and pneumonia status may hold diagnostic potential, due to the close anatomical approximation of the oral cavity with the lungs and gastrointestinal tract, and the interface formed with the external environment. Given the poor reliability of sampling the infected lung ${ }^{7}$, which must be performed essentially 'blind', the ease of access to the oropharynx for microbial sampling could lead to rapid, reliable identification of potential causative microorganisms, and provide antimicrobial susceptibility profiles to aid diagnosis and treatment of pneumonia ${ }^{31}$.

Recruitment of eligible participants was a major challenge encountered during this study as many care home residents were cognitively impaired and thus unable to consent. Similarly, a number of pneumonia patients had cognitive impairment either as a background comorbidity or due to acute delirium. The cross-sectional design of this study was another limitation. As recruited respiratory ward patients had received a diagnosis of pneumonia prior to recruitment, it was not possible to track changes in composition of the oral microbiota from respiratory health to disease. Similarly, there was no follow-up to examine shifts in microbial communities upon resolution of pneumonia. It was therefore not possible to determine if changes in the oral microbiome preceded pneumonia onset, a key step in determining causality ${ }^{32}$.

All patients with suspected pneumonia received empirical antibiotic therapy according to local policy, which reflects the British Thoracic Society guidelines on the management of severe community acquired pneumonia ${ }^{33}$. As only a low proportion of care home residents had received any antimicrobials in the preceding 30 days, differential antibiotic use is a potential confounder for the altered oral microbial composition seen. However, a number of factors suggest that while antibiotic use may have contributed to reduced community diversity and species richness, the differences cannot be entirely explained by antibiotic use alone. Firstly, it would be expected that denture-associated biofilms would be least affected by antibiotic use compared with other oral sites, as biofilms may confer antimicrobial tolerance to constituent microbes ${ }^{34}$. Moreover, antibiotics have to traverse the oral mucosal barrier, diffuse through the palatal microbial biofilm and then penetrate the denture-associated biofilm in sufficient concentration to perturb microbial communities.

It should be noted that Enterobacteriaceae are typically not susceptible to macrolide antibiotics such as clarithromycin and are intrinsically resistant to amoxicillin and other beta-lactamases ${ }^{35}$. The aggressive use of these antibiotic regimes in pneumonia patients may act as a selective pressure to suppress growth and survival of normal oral microbes, particularly Streptococcaceae, leading to an increased relative abundance of more virulent microorganisms ${ }^{36}$. Nonetheless, the finding that the difference in relative abundance of PRPs between cohorts was most pronounced in denture samples suggests that antibiotic use was unlikely to be the primary contributor to the changes in microbial community composition. Notably, no S. aureus isolates recovered from respiratory ward patients were resistant to amoxicillin, compared to over one quarter of those from care home residents. However, macrolide resistance was more than doubled in respiratory ward S. aureus isolates. There were much higher rates of resistance to the beta-lactam antibiotic piperacillin-tazobactam in P. aeruginosa isolates from care home residents compared with pneumonia patients, as was seen for the related cephalosporin ceftazidime. However, resistance of P. aeruginosa isolates to ciprofloxacin, a fluoroquinolone antibiotic, was found to be much higher among pneumonia patients than care home residents. While the low number of both S. aureus and P. aeruginosa isolates 
recovered precludes any reliable statistical evaluation, the equivocal resistance patterns observed suggest that antibiotic treatment may not have exerted a major selective pressure upon the oral microbiota. This was particularly evident in the case of S. aureus isolates, where amoxicillin sensitive strains were isolated from respiratory ward patients' samples despite empiric therapy with this agent.

\section{Conclusions}

This study revealed that perturbations within the denture-associated oral microbiome are associated with pneumonia. Having demonstrated an association between a deranged oral microbiome and an increase in the bioburden of putative respiratory pathogens, the premise for a causal association is established. Salivary levels of IL-6 and IL-8 were correlated with the oral bioburden of putative respiratory pathogens, and so may hold potential for identifying individuals heavily colonised by such microorganisms and thus at risk of respiratory infection. Future research should aim to further disentangle the relationship between oral health, the oral microbiome and pneumonia pathogenesis; as well as assessing the impact of effective oral and denture care on modulating the oral microbiome and decreasing pneumonia risk in susceptible individuals.

\section{Methods}

\section{Participant recruitment and sample collection}

We recruited a total of 66 denture-wearing individuals from long term residential care facilities $(n=35)$ and hospital wards $(n$ = 26). Participants were excluded from either group if they lacked capacity to provide consent; were receiving palliative endof-life care; had taken part in another study in the preceding 6 months; were severely immunosuppressed, immunocompromised; or had a diagnosis of oro-pharyngeal or lung malignancy. Care home residents were excluded if they had a history of respiratory infection in the previous 30 days. Hospitalised patients were included only if there was a confirmed diagnosis of pneumonia supported by radiographic signs. Where this information was not readily available, a diagnosis was sought from the treating respiratory physician.

For each participant, a brief dental history and examination was undertaken; including denture cleaning habits, oral mucosal inflammation (assessed by Newton's inde ${ }^{20}$ ) and a record of decayed, missing and filled teeth ${ }^{21}$ as a surrogate marker of previous oral disease burden. Imprint cultures were taken from the dorsal tongue, denture-bearing palatal mucosa and denture-fit surface of each participant, and transferred sequentially to CHROMagar ${ }^{\circledR}$ (CHROMagar Microbiology Company, Paris, France), Mannitol Salt agar (Lab M, Heywood, UK) and Pseudomonas agar (Lab M, Heywood, UK) for 60 s each. Sterile cotton swabs were taken from the same sites using a standardised technique and transferred to Amies transport medium. A sterile cotton salivette was placed in the buccal sulcus to collect unstimulated saliva over $1 \mathrm{~min}$.

Agar plates were incubated aerobically at $37^{\circ} \mathrm{C}$ for $24-72 \mathrm{~h}$ until distinct colonies could be identified. Cultured microorganisms were characterised by routine histological staining and biochemical testing to differentiate Candida albicans, Staphylococcus aureus and Pseudomonas aeruginosa. The antimicrobial susceptibility of $S$. aureus and $P$. aeruginosa isolates was tested according to the EUCAST disc-diffusion method ${ }^{22}$.

Salivettes were centrifuged twice at $3000 \times \mathrm{g}$ to recover decellularised saliva, from which a panel of pro-inflammatory cytokines were subsequently analysed using a Cytometric Bead Array kit (BD Biosciences, Wokingham, UK) and flow-assisted cell sorting (BD FACSCanto II, BD Biosciences, Wokingham, UK). Cytokine profiles were assessed according to pneumonia status and relative abundance of denture-associated potential respiratory pathogens. 


\section{Bacterial DNA extraction from oral and denture samples, and detection of Streptococcus pneumoniae by qPCR and 16S rRNA gene sequencing}

Microbial swabs were aseptically transferred to $10 \mathrm{ml}$ bijou bottles containing $1 \mathrm{ml}$ of $0.9 \%$ PBS by cutting the swab neck with flame-heated scissors. DNA extraction was performed using the Qiagen PuraGene kit (Qiagen, Manchester, UK) using the protocol for Gram-positive bacteria, with a final elution volume of $20 \mathrm{ml}$. The following modifications were added to this protocol: bijou bottles containing microbial swabs were vortexed at high speed for $1 \mathrm{~min}$, and the resultant cell suspension transferred by pipetting to a $1.5 \mathrm{ml}$ microcentrifuge tube on ice. Cell suspensions were centrifuged for $1 \mathrm{~min}$ at $5000 \mathrm{xg}$ and the supernatant discarded by pouring. The resultant cell pellet was then resuspended in $1 \mathrm{ml}$ of Qiagen PuraGene cell suspension solution (Qiagen, Manchester, UK).

Due to the challenges associated with speciating S. pneumoniae by 16S rRNA gene sequencing, a species-specific TaqManä assay which targeted the autolysin-encoding gene lytA was used for detection of this microorganism by qPCR. The primers used in this assay were:

Forward primer sequence: ACGCAATCTAGCAGATGAAGCA,

Reverse primer sequence: TCGTGCGTTTTAATTCCAGCT,

Probe sequence: YY-TGCCGAAAACGCTTGATACAGGGAG-BHQ1

This assay had previously been published as part of a multiplex diagnostic assay ${ }^{23}$. The sensitivity and specificity of the assay in single-plex use was confirmed by standard curve, using reference strains S. pneumoniae ATCC 49619, Streptococcus gordonii ATCC 10558 and Streptococcus sanguinis ATCC 7863 in 10-fold serial dilutions to a lower limit of approximately 10 cells/ml. PCR was undertaken in triplicate using a QuantStudio 6 Flex instrument (Applied Biosystemsä, California, USA).

Library preparation and sequencing was undertaken by Research and Testing Laboratories (RTL, Texas, USA) using the Illumina Miseq $28 f$ and 519r primers to overlap the V1 - V3 hypervariable regions of the 16S rRNA gene. A two-step amplification process was used with a preamplification step employing the Illiumina i5 and i7 primers initially Sequencing parameters and primer sequences used can be found in the supporting information.

\section{S rRNA gene sequence pre-processing}

Sequencing data was provided as paired FASTQ files for each sample. Generation of 16S rRNA gene sequences was undertaken using the open-source software MOTHUR ${ }^{24}$. The Illumina MiSeq standard operating procedure was followed throughout. Paired end reads were first assembled with the make.contigs command. This command combines the data from the paired FASTQ files and provides a quality score for each file. Each contig was then filtered using the screen.seqs command, using the parameters: $\operatorname{maxn}=0$, maxambig $=0$, maxhomop $=5$, maxlength $=605$. Reads were subsampled to 675 which resulted in the exclusion of 3 samples ( 2 from pneumonia patients, 1 from a care home resident).

Rare operational taxonomic units OTUs (<10 reads) were excluded from further analysis and any OTUs with less than $98 \%$ coverage or $97 \%$ sequence identity to a known bacterial species were categorised to genus level only. After manual scanning, OTUs that would not be expected to occur in the oral cavity were re-examined using the NCBI BLASTn database.

\section{Statistical analyses}

Statistical analysis was conducted using $\mathrm{R}^{25}$, SPSS 21, Graphpad Prism 8.0 and Microsoft Excel. Simple descriptive summary statistics were generated for participant demographic data and oral health measures. Age, Charlson Index ${ }^{26}$, 
Denture Hygiene Score, Newton's Classification ${ }^{20}$ and DMFT $^{21}$ scores were treated as continuous variables. The remaining data were analysed as categorical variables. Distribution of data was assessed by visual inspection of histograms, the Kolgomorov-Smirnov test of normality (alpha set to $\mathrm{p}<0.05$ ) and inspection of Q-Q plots. To assess differences between participant cohorts at baseline, the Kruskal-Wallis test was undertaken on nonparametric data, while two-way ANOVA with Bonferroni correction was used to analyse normally distributed continuous data. Categorical variables were analysed using the Chi-Squared ( goodness of fit test.

Alpha diversity was measured by the Chao2 and Inverse Simpson Indices. Alpha diversity indices were compared using the Kruskal-Wallis test and Median K-tests.

Multivariate logistic regression was employed to assess the impact of demographic and oral health variables on Chao and Inverse Simpson indices using a forward stepwise approach.

PRP species were assigned to 9 groupings: enterococci, Acinetobacter spp. Enterobacteriaceae, Haemophilus spp., Klebsiella spp., P. aeruginosa, Serratia spp., S. aureus, and Escherichia coli. The percentage relative abundance of PRP species was calculated and analysed using the Two-Stage Linear Step up Procedure of Benjamini, Krieger and Yekutieli ${ }^{27}$ to control the false discovery rate, with Q-value set at 0.05 . Fold differences between participant cohorts' PRP relative abundance were calculated for each oral site.

Percentage relative abundance was converted to decimal data, and Linear discriminant analysis of Effect Size (LEfSe) conducted using the open access galaxy module ${ }^{28}$.

Statistical analysis of salivary cytokine profiles was undertaken using Microsoft Excel and Graphpad Prism 8.0. Normality was assessed using the Kolgomorov-Smirnov test with an alpha of 0.05. Subsequent analysis was undertaken using MannWhitney $U$ tests with Bonferroni correction applied post-hoc. The significance threshold selected for Bonferroni-adjusted test results was $\mathrm{p}<0.05$.

\section{Abbreviations}

- CAP

- community acquired pneumonia

- DMFT

- decayed, missing and filled teeth

- HAP

- hospital acquired pneumonia

- OTU

- operational taxonomic unit

- PRP

- putative respiratory pathogen

- VAP

- ventilator associated pneumonia

\section{Declarations}

\section{Ethics declarations}

Ethical approval for this study was obtained from the Wales REC 6; reference 16/WA/0317. All participants provided written consent for this study.

\section{Consent for publication}

Page 10/19 
Not applicable

\section{Availability of supporting data and materials}

The 16S rRNA gene sequences, associated datasets and metadata generated for this study are available through the Open Science Framework online repository: [https://osf.io/mknsu/]

\section{Competing interests}

The authors declare that they have no competing interests

\section{Funding}

This research was funded by Cardiff University as part of the completion of a PhD for JAT. Additional funding was awarded by the Oral and Dental Research Trust. Neither funding body had any input into the study design, conduct, analysis or in writing the manuscript.

\section{Authors' contributions}

JAT, MJW, JL, MW and DWW contributed to the study concept and design, providing technical input and guidance throughout the study. JAT and AS analysed and interpreted the study data. JAT and $\mathrm{CH}$ recruited sites and participants for the study. JAT was primarily responsible for writing the manuscript, with major input from all authors. All authors read and approved the final manuscript.

\section{Acknowledgements}

We would like to thank the Oral and Dental Research Trust, who provided funding for part of this work. We would also like to thank the many staff members at University Hospital Wales, University Hospital Llandough and the care homes visited who generously gave their time and assistance for this study. Finally, we would like to thank Mandy Wootton and colleagues for their input and expertise regarding identification of Streptococcus pneumoniae, and for kindly providing reference strains used in this study.

\section{Additional information}

None.

\section{References}

1. Lim SS, Vos T, Flaxman AD et al. A comparative risk assessment of burden of disease and injury attributable to 67 risk factors and risk factor clusters in 21 regions, 1990-2010: a systematic analysis for the Global Burden of Disease Study 2010. The Lancet. 2013;380(9859):2224-60.

2. Welte T, Torres A, Nathwani D. Clinical and economic burden of community-acquired pneumonia among adults in Europe. 2012;67(1):71-9.

3. Myles PR, Hubbard RB, Gibson JE, Pogson Z, Smith CJ, McKeever TM. Pneumonia mortality in a UK general practice population cohort. The European Journal of Public Health. 2009;19(5):521-6.

4. McLuckie A. Respiratory disease and its management. Springer Science \& Business Media; 2009.

5. Cillóniz C, Civljak R, Nicolini A, Torres A. Polymicrobial community-acquired pneumonia: an emerging entity. Respirology. 2016;21(1):65-75. 
6. Torres A, Blasi F, Peetermans WE, Viegi G, Welte T. The aetiology and antibiotic management of community-acquired pneumonia in adults in Europe: a literature review. European journal of clinical microbiology \& infectious diseases. 2014;33(7):1065-79.

7. Torres A, Lee N, Cillóniz C, Vila J, Van der Eerden M. Laboratory diagnosis of pneumonia in the molecular age. European Respiratory Journal. 2016;48(6):1764-78.

8. Azarpazhooh A, Leake JL. Systematic review of the association between respiratory diseases and oral health. Journal of periodontology. 2006;77(9):1465-82.

9. O'Grady NP, Murray PR, Ames N. Preventing ventilator-associated pneumonia: does the evidence support the practice? Jama. 2012:307(23):2534-9.

10. Paju S, Scannapieco FA. Oral biofilms, periodontitis, and pulmonary infections. Oral diseases. 2007;13(6):pp.508-12.

11. Awano S, Ansai T, Takata Y, Soh I, Akifusa S, Hamasaki T, Yoshida A, Sonoki K, Fujisawa K, Takehara T. Oral health and mortality risk from pneumonia in the elderly. Journal of dental research. 2008;87(4):334-9.

12. Sands KM, Twigg JA, Lewis MA, Wise MP, Marchesi JR, Smith A, Wilson MJ, Williams DW. Microbial profiling of dental plaque from mechanically ventilated patients. Journal of medical microbiology. 2016;65(Pt 2).

13. Sands KM, Wilson MJ, Lewis MA, Wise MP, Palmer N, Hayes AJ, Barnes RA, Williams DW. Respiratory pathogen colonization of dental plaque, the lower airways, and endotracheal tube biofilms during mechanical ventilation.Journal of critical care. 2017;37:30-7.

14. Hua F, Xie H, Worthington HV, Furness S, Zhang Q, Li C. Oral hygiene care for critically ill patients to prevent ventilatorassociated pneumonia. Cochrane Database of Systematic Reviews. 2016(10).

15. Russell SL, Boylan RJ, Kaslick RS, Scannapieco FA, Katz RV. Respiratory pathogen colonization of the dental plaque of institutionalized elders. Special care in dentistry. 1999;19(3):128-34.

16. Sumi Y, Miura H, Michiwaki Y, Nagaosa S, Nagaya M. Colonization of dental plaque by respiratory pathogens in dependent elderly. Archives of gerontology and geriatrics. 2007;44(2):119-24.

17. O'Donnell LE, Smith K, Williams C, Nile CJ, Lappin DF, Bradshaw D, Lambert M, Robertson DP, Bagg J, Hannah V, Ramage G. Dentures are a reservoir for respiratory pathogens. Journal of Prosthodontics. 2016;25(2):99-104.

18. Yoneyama T, Yoshida M, Ohrui T, Mukaiyama H, Okamoto H, Hoshiba K, Ihara S, Yanagisawa S, Ariumi S, Morita T, Mizuno Y. Oral care reduces pneumonia in older patients in nursing homes. Journal of the American Geriatrics Society. 2002;50(3):430-3.

19. Scannapieco FA, Binkley CJ. Modest reduction in risk for ventilator-associated pneumonia in critically ill patients receiving mechanical ventilation following topical oral chlorhexidine. Journal of Evidence Based Dental Practice. 2012;12(2):103-6.

20. Newton AV. Denture sore mouth: a possible aetiology. Br Dent J. 1962;112:357-360.

21. Klein H, Palmer CE, Knutson JW. Studies on dental caries. Public health rep. 1938;53:751-65.

22. The European Committee on Antimicrobial Susceptibility Testing Disc diffusion manual v6.0. 2017. http://www.eucast.org/ast_of_bacteria/disk_diffusion_methodology/

23. Gadsby NJ, McHugh MP, Russell CD, Mark H, Morris AC, Laurenson IF, Hill AT, Templeton KE. Development of two realtime multiplex PCR assays for the detection and quantification of eight key bacterial pathogens in lower respiratory tract infections. Clinical microbiology and infection. 2015;21(8):788-91.

24. R Core Team. R: A language and environment for statistical computing. 2013.

25. Schloss PD, Westcott SL, Ryabin T, Hall JR, Hartmann M, Hollister EB, Lesniewski RA, Oakley BB, Parks DH, Robinson CJ, Sahl JW. Introducing mothur: open-source, platform-independent, community-supported software for describing and comparing microbial communities. Appl. Environ. Microbiol.. 2009;75(23):7537-41.

26. Charlson ME, Pompei P, Ales KL, MacKenzie CR. A new method of classifying prognostic comorbidity in longitudinal studies: development and validation. Journal of chronic diseases. 1987;40(5):373-83. 
27. Benjamini Y, Krieger A, Yekutieli D. Two staged linear step up FDR controlling procedure. Tel Aviv University, and Department of Statistics, Wharton School, University of Pennsylvania. 2001.

28. Segata N, Izard J, Waldron L, Gevers D, Miropolsky L, Garrett WS, Huttenhower C. Metagenomic biomarker discovery and explanation. Genome biology. 2011;12(6):R60.

29. Zomorodian K, Haghighi NN, Rajaee N, Pakshir K, Tarazooie B, Vojdani M, Sedaghat F, Vosoghi M. Assessment of Candida species colonization and denture-related stomatitis in complete denture wearers. Medical mycology. 2011;49(2):208-211.

30. Dickson RP, Huffnagle GB. The lung microbiome: new principles for respiratory bacteriology in health and disease. PLoS pathogens. 2015;11(7):e1004923.

31. Holter JC, Müller F, Bjørang O, Samdal HH, Marthinsen JB, Jenum PA, Ueland T, Frøland SS, Aukrust P, Husebye E, Heggelund L. Etiology of community-acquired pneumonia and diagnostic yields of microbiological methods: a 3-year prospective study in Norway. BMC infectious diseases. 2015;15(1):64.

32. Hill AB. The Environment and Disease: Association or Causation. Proceedings of the Royal Society of Medicine. 1965;58:295-300.

33. Lim WS, Baudouin SV, George RC, Hill AT, Jamieson C, Le Jeune I, Macfarlane JT, Read RC, Roberts HJ, Levy ML, Wani M. BTS guidelines for the management of community acquired pneumonia in adults: update 2009. Thorax. 2009;64(Suppl 3):iii1-55.

34. Stewart PS. Antimicrobial tolerance in biofilms. Microbiology spectrum. 2015;3(3).

35. Bouza E and Cercenado E., September. Klebsiella and enterobacter: antibiotic resistance and treatment implications. Seminars in respiratory infections. 2002;17(3):215-30.

36. Sommer MO, Dantas G. Antibiotics and the resistant microbiome. Current opinion in microbiology. 2011;14(5):556-563.

\section{Figures}




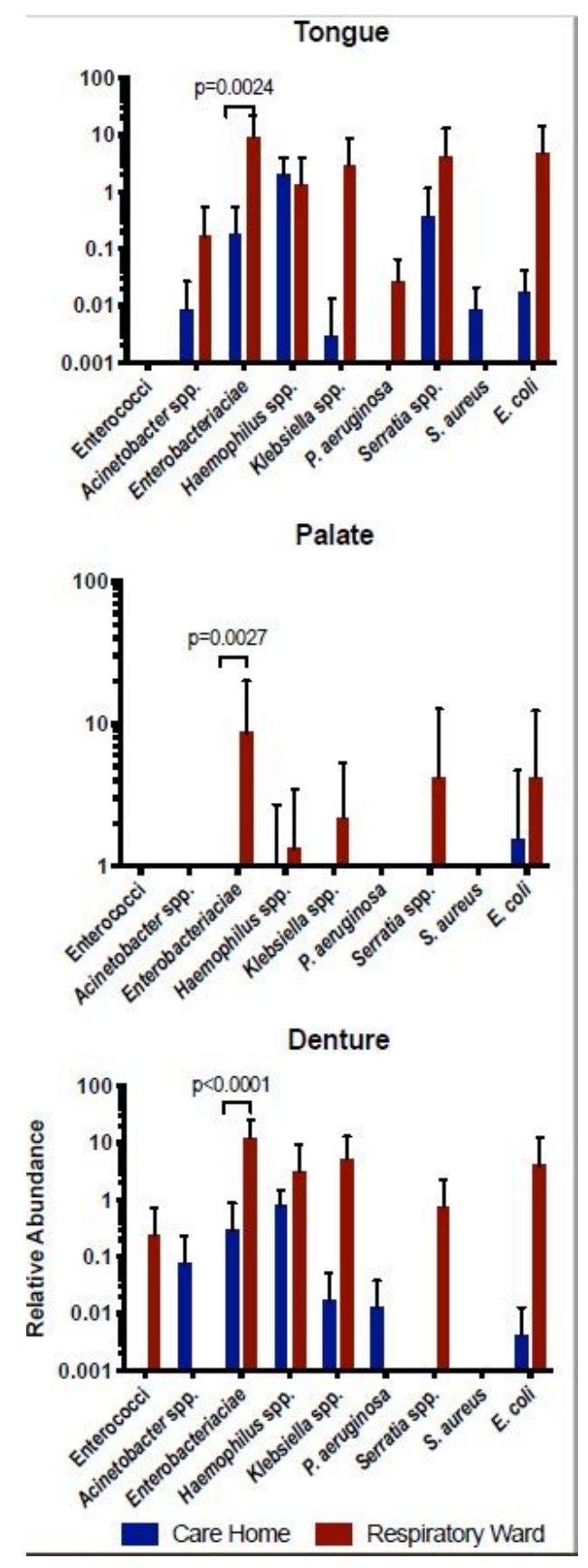

Figure 1

Relative abundance (\%) of putative respiratory pathogens from each oral site OTUs are grouped at either genus or species level to collate bacteria associated with respiratory infection at the lowest discriminatory phylogenetic level. Note that the $Y$ axis features a log10 scale. Mean values shown, error bars represent $95 \%$ confidence intervals. 

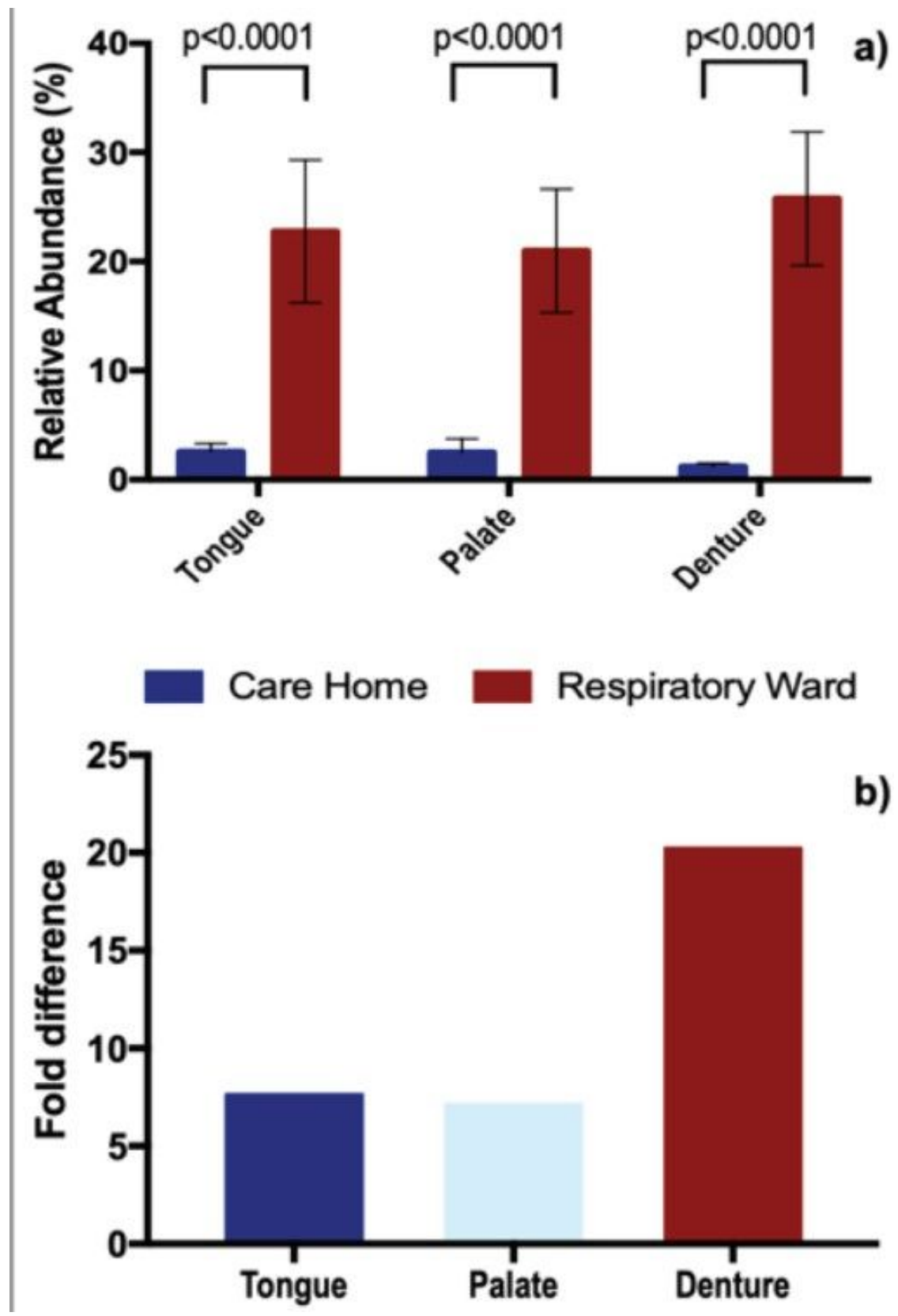

Figure 2

a) - Cumulative relative abundance (\%) of PRPs identified in care home residents compared to respiratory ward patients. Mean values shown. Error bars represent 95\% confidence intervals. b) - Fold difference of PRP cumulative relative abundance in respiratory ward patients, normalised to care home residents. Fold difference calculated using mean values reported in a). 

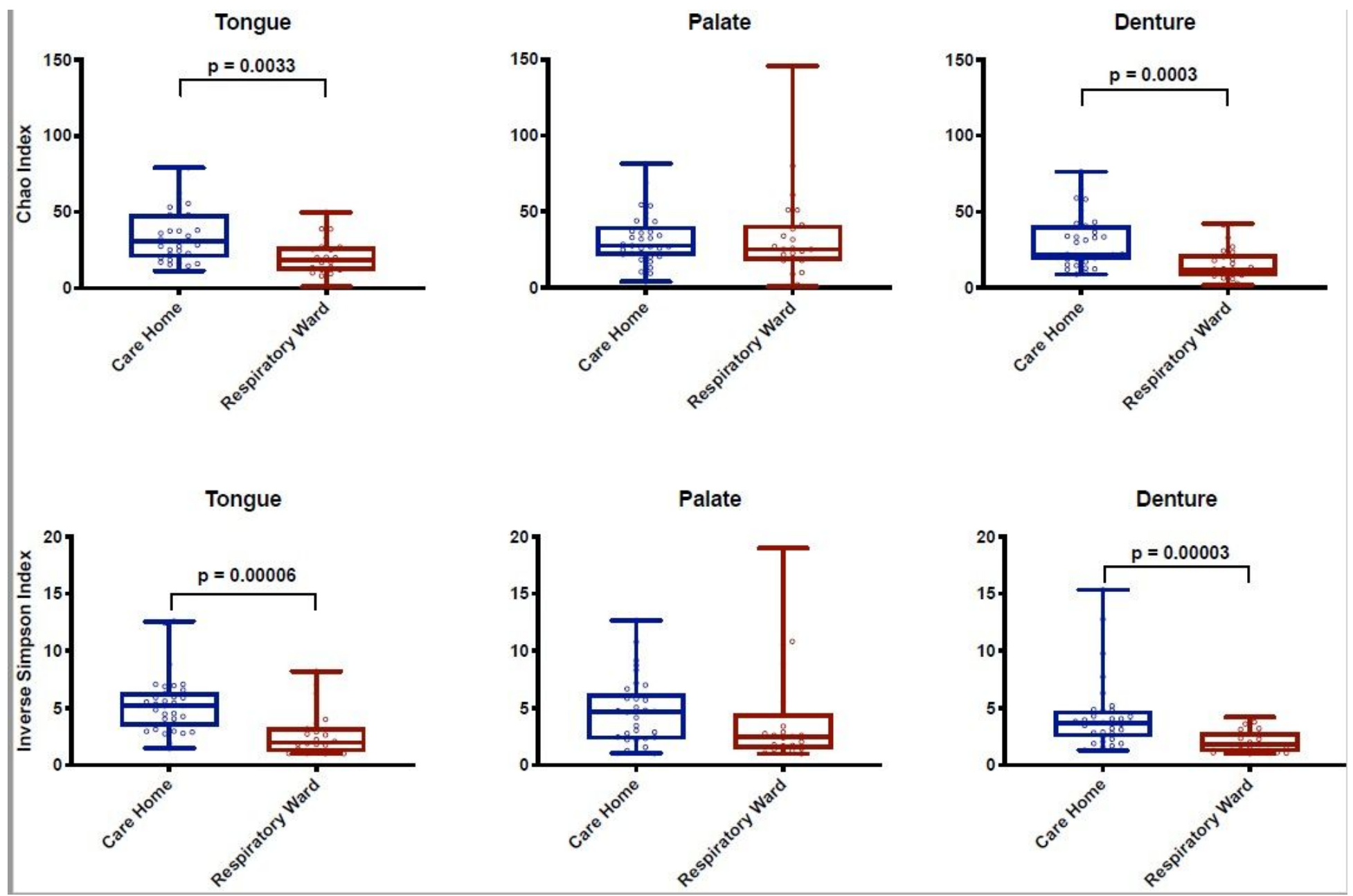

Figure 3

Chao (upper panel) and Inverse Simpson (lower panel) indices for oral sites in each participant cohort Individual data points with representative box plots shown. 
a)

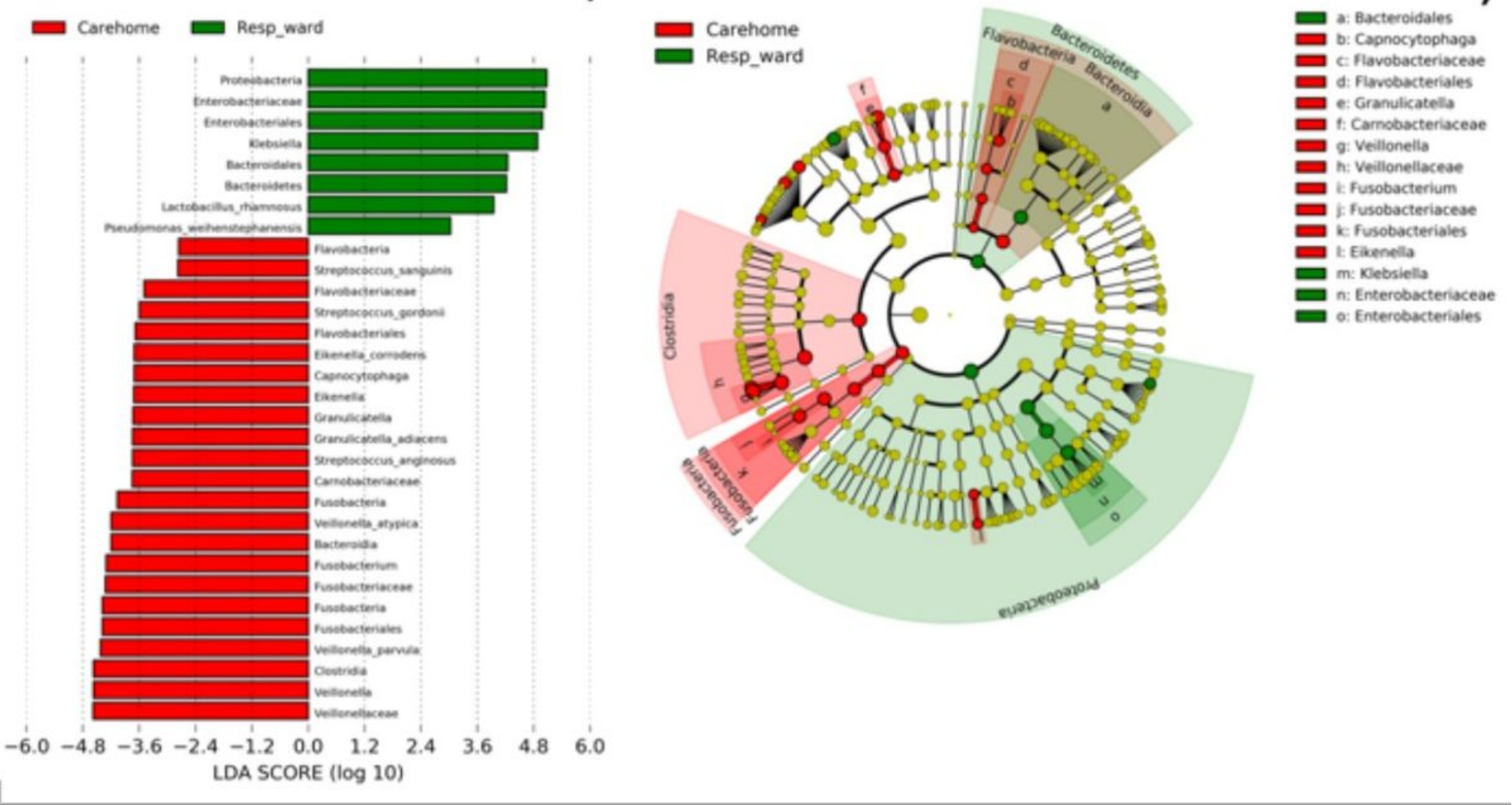

\section{Figure 4}

LeFSe analysis of differential OTU relative abundance between participant cohorts for denture samples a) Histogram of significantly different Linear Discriminant Analysis scores for samples. b) Cladogram representing taxonomic relationships of significantly different abundances between cohort samples. Green indicates taxons with increased abundance in pneumonia patients, red indicates taxons with increased abundance in samples from care home residents. Yellow circles represent taxa which showed no significant differences between cohorts. The diameter of each circle in the cladogram is proportional to the relative abundance of the taxon represented. 


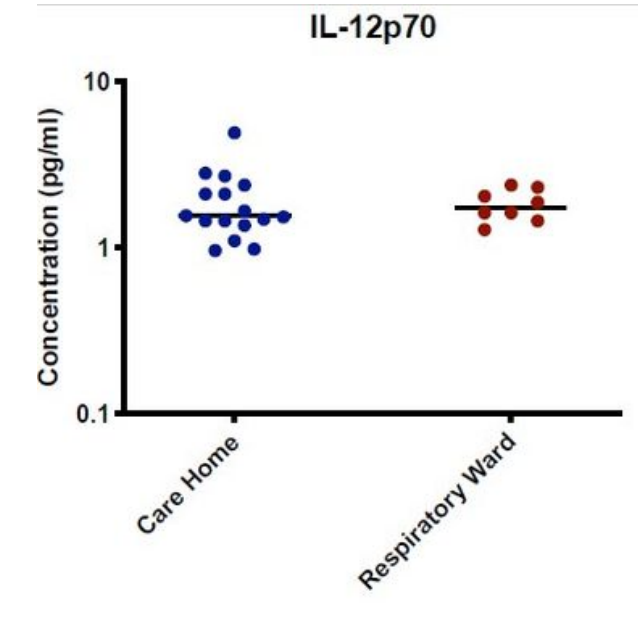

IL-1B

IL-6
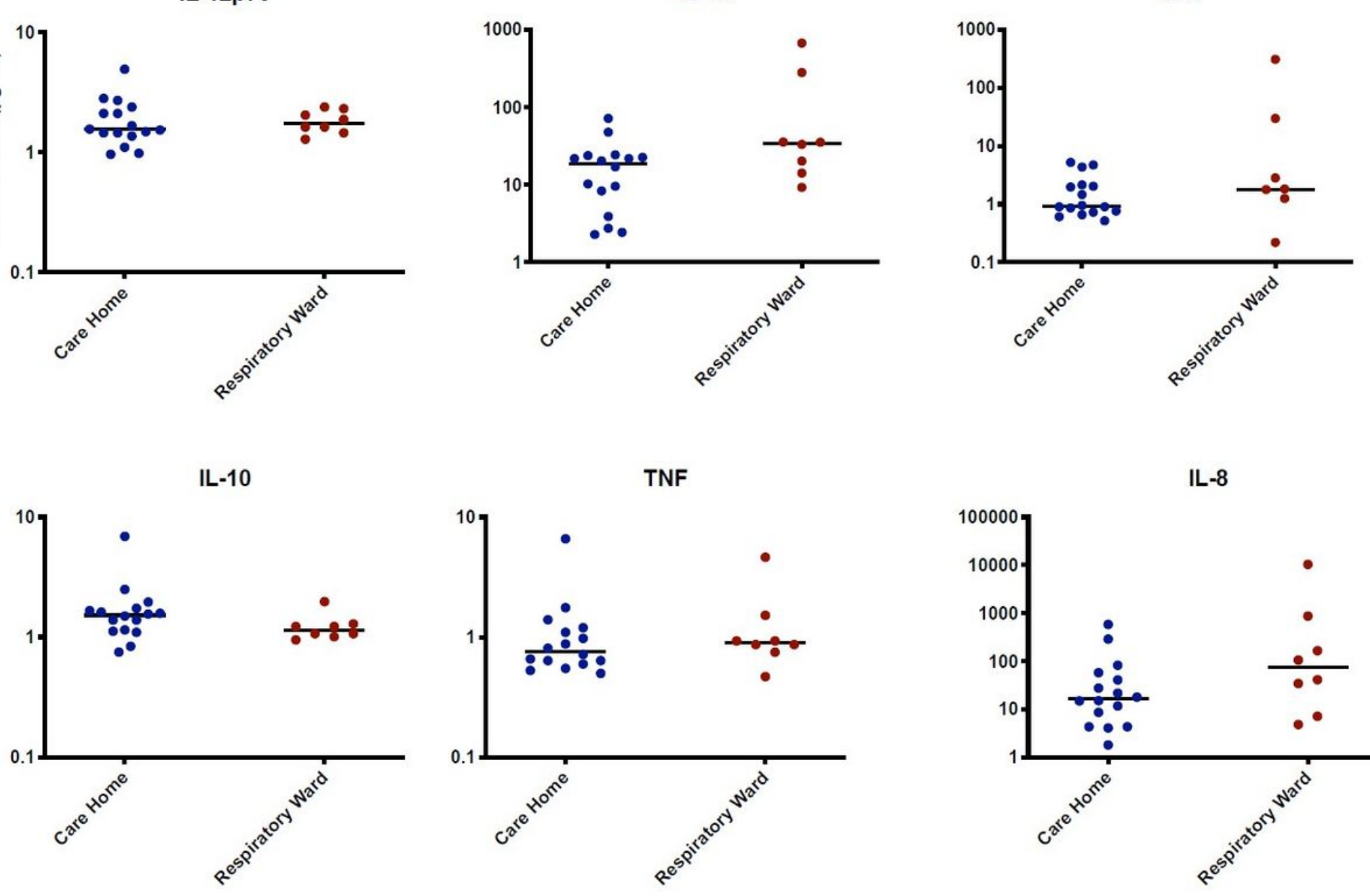

IL-8

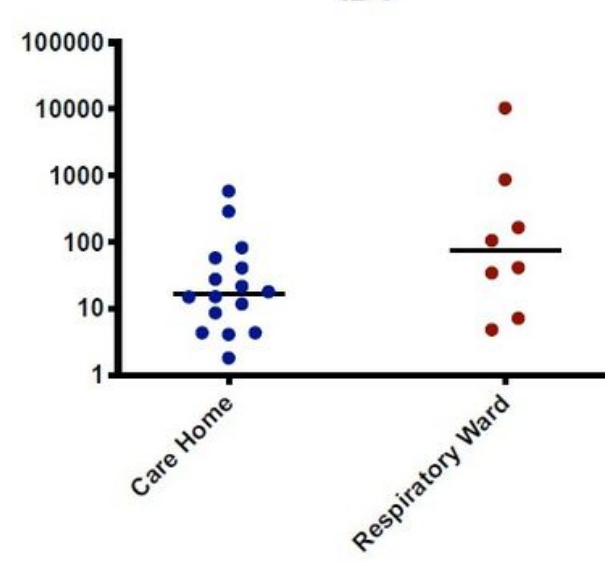

Figure 5

Salivary cytokine expression levels $(\mathrm{pg} / \mathrm{ml})$ Note that the $\mathrm{Y}$ axis uses a log10 scale. Individual points represent each sample measured. Horizontal black line indicates median expression level for each cohort. 


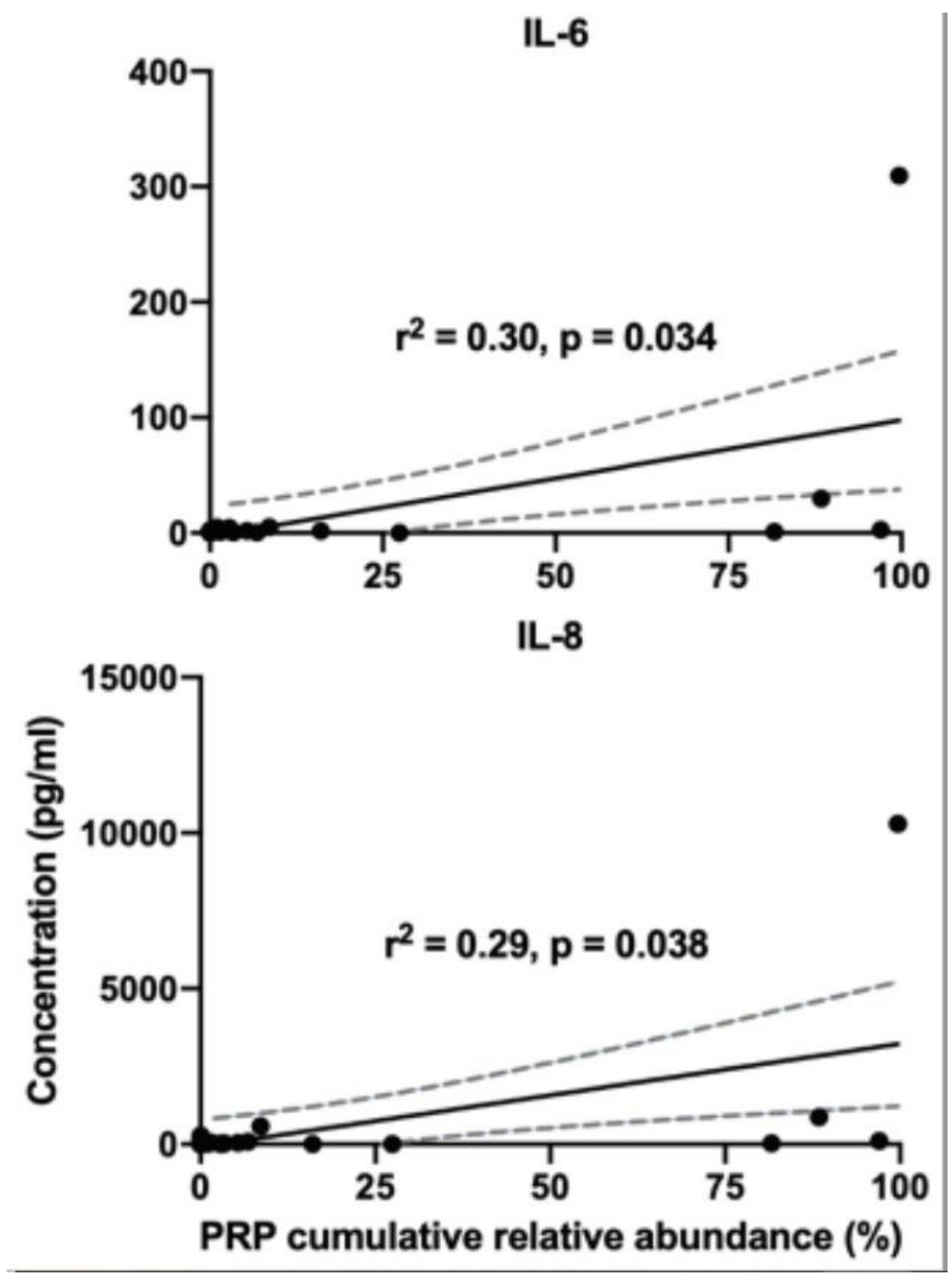

Figure 6

Linear regression of salivary cytokine level against cumulative relative abundance of PRP species $95 \%$ confidence intervals indicated by dashed lines. P values represent significance following $\mathrm{F}$ test with Bonferroni post-hoc correction. 